\title{
Erreichbarkeitsmanagement im Unternehmen
}

\section{Schaffung unterbrechungsfreier Regenerationsphasen durch organisatorische und technische Maßnahmen zur Einschränkung ständiger Erreichbarkeit}

Daniel Grießhaber (D), Uwe Laufs, Johannes Maucher, Nadine Miedzianowski, Zofia Saternus (1) und Katharina Staab

\subsection{Motivation und Projektziele}

Durch die heute umfangreiche Verbreitung des Internets und des Mobilfunks prägen und verändern Informations- und Kommunikationstechnologien (IKT) das Arbeitsumfeld erheblich [13, 15]. Smartphones und Laptops führen dazu, dass Beschäftigte ständig erreichbar sind und auch arbeiten können, unabhängig von Ort und Zeit. Nicht nur Arbeitsleistung und Produktivitätsdruck erhöhen sich durch die Nutzung moderner IKT, ebenso wird die Trennung zwischen Beruf und Privatleben sowohl in zeitlicher als auch räumlicher Hinsicht zunehmend aufgehoben [2, 3]. Angesichts steigender Fehltage aufgrund psychisch verursachter Erkrankungen [1], fürchten ins-

D. Grießhaber · J. Maucher

Hochschule der Medien, Stuttgart, Deutschland

U. Laufs $(\bowtie)$

Fraunhofer IAO, Stuttgart, Deutschland

N. Miedzianowski

Universität Kassel, Projektgruppe Verfassungsverträgliche Technikgestaltung (provet),

Kassel, Deutschland

Z. Saternus

Goethe Universität Frankfurt, Frankfurt am Main, Deutschland

K. Staab

Technische Universität Darmstadt Fachgebiet Marketing \& Personalmanagement,

Darmstadt, Deutschland

(C) Der/die Autor(en) 2021

W. Bauer et al. (Hrsg.), Arbeit in der digitalisierten Welt,

https://doi.org/10.1007/978-3-662-62215-5_23 
besondere Arbeitnehmervertreter und Politiker negative gesundheitliche Folgen für Beschäftigte aufgrund zunehmender Entgrenzung von Privat- und Berufsleben durch permanente technologische Erreichbarkeit. Es ist $\mathrm{zu}$ beobachten, dass eine steigende Anzahl an Unternehmen nach Lösungen im Sinne eines gesundheitsfördernden und effektiven Erreichbarkeitsmanagements für ihre Beschäftigten sucht. Aktuell reichen diese von Schulungen der Mitarbeiter bis hin zu „digitalen Sperrstunden“, z. B. durch das Abschalten des E-Mail-Servers nach Feierabend. Vielfach bieten diese Lösungen allerdings eher globale Lösungen, ohne die individuellen Bedürfnisse der Beschäftigten zu berücksichtigen. So deuten beispielsweise die Ergebnisse einer qualitativen Studie von Stock-Homburg et al. [14] darauf hin, dass für Führungskräfte bzgl. unterschiedlicher Lösungen im Bereich des Erreichbarkeitsmanagements größerer Bedarf besteht als für Mitarbeitende ohne Führungsverantwortung. Darüber hinaus spielt der Grad der Internationalisierung der Tätigkeit eine Rolle für die Gestaltung des Erreichbarkeitsmanagements. Das Forschungsprojekt SANDRA adressiert das betriebliche Erreichbarkeitsmanagement sowohl mit organisatorischen Maßnahmen als auch mit einer technischen Lösung, einem Erreichbarkeitsassistenten für Smartphones, der E-Mails ggf. intelligent verzögert beziehungsweise Anrufe blockt, um unterbrechungsfreie Regenerationspausen für Beschäftigte zu schaffen [4].

Die Wirksamkeit bei der Stressminderung wird mit einem im Leistungssport bereits erfolgreich eingesetzten Verfahren geprüft. Neben der Einbeziehung aller wichtigen Interessengruppen stehen vor allem die datenschutzrechtlichen sowie die arbeitsrechtlichen Erfordernisse im Fokus. Diese fließen von Beginn an in die Ausgestaltung der Arbeit und die Entwicklung von Werkzeugen und Methoden ein.

\subsection{Ergebnisse}

\subsubsection{Rechtliche Anforderungen an ein betriebliches Erreichbarkeitsmanagement}

Die Entwicklung und der Einsatz eines Erreichbarkeitsmanagement-Systems (EMS) im Arbeitskontext geht mit unterschiedlichen rechtlichen Fragestellungen einher: Wie ist das System zu gestalten, um eine rechtskonforme Anwendung zu gewährleisten und welche rechtlichen Vorgaben sind einzuhalten? Welche Chancen und Risiken können aus der Verwendung einer solchen Technologie im Arbeitskontext prognostiziert werden und welche rechtlichen und technischen Anforderungen ergeben sich daraus für die Technikgestaltung?

Damit das EMS die Erreichbarkeit von Beschäftigten regulieren kann, benötigt es eine große Menge an Daten über seine Nutzer. Zum einen fallen bereits durch den Systemgebrauch verschiedene Daten, wie Inhaltsdaten aus beispielsweise eingehenden E-Mails oder Kalendereinträgen, an. Zum anderen hinterlegen die Beschäftigten im System selbstständig Informationen über ihre Erreichbarkeitssituation (z. B. Beginn und 
Ende der Arbeitszeit). Auf diese und weitere Informationen greift das System zur Entscheidungsfindung und Steuerung eingehender Kommunikationsversuche zu. Hieraus ergeben sich unterschiedliche Risiken für die Nutzer sowie den Arbeitgeber, die zunächst im Projekt identifiziert wurden (s. hierzu [6, 7]). Mithilfe des technischen Systems und den bei der Systemnutzung anfallenden Daten ist es z. B. dem Arbeitgeber oder Dritten potenziell möglich, dieses zur Überwachung der Beschäftigten zu missbrauchen und die Daten zweckentfremdet zu nutzen. mitilfe der Daten könnten ein Bewegungs-, Kommunikations- und Persönlichkeitsprofil über den Systemnutzer erstellt werden. Aus der möglichen Überwachung könnten ferner Informationen zur Anwesenheits-, Leistungs- und Verhaltenskontrolle herangezogen werden. An dieser Stelle ist jedoch anzumerken, dass ein System zur Verbesserung der beruflichen Erreichbarkeitssituation vor allem durch solche Arbeitgeber eingesetzt wird, die gerade kein Kontrollinteresse gegenüber ihren Mitarbeitern haben, sodass insbesondere moderne Arbeitgeber, deren Unternehmen durch dynamische und vertrauensvolle Arbeitsstrukturen gekennzeichnet sind, ein EMS in ihren Unternehmen einsetzen möchten. Trotzdem ist es grundsätzlich geeignet zur Kontrolle und Überwachung der Systemnutzer verwendet zu werden. Dies ist jedoch stets von den Zugriffsmöglichkeiten des Arbeitgebers und Dritter auf das System abhängig.

Aufgrund der Verarbeitung von unterschiedlichen Daten ist zum einen das Datenschutzrecht - insbesondere die Bestimmungen der Datenschutz-Grundverordnung (DSGVO) und des Bundesdatenschutzgesetzes (BDSG) - bei der Gestaltung des EMS zu berücksichtigen. Grundsätzlich müssen personenbezogene Daten gemäß Art. 5 Abs. 1 lit. a Var. 1 DSGVO auf rechtmäßige Weise verarbeitet werden. Daraus folgt, dass das EMS nur auf der Grundlage einer Einwilligung i. S. v. Art. 6 Abs. 1 Satz 1 lit. a DSGVO oder gesetzlichen Ermächtigung nach lit. b oder lit. c personenbezogene Daten verarbeiten darf. Daneben ergeben sich weitere Erlaubnistatbestände gemäß Art. 9 Abs. 2 und 88 DSGVO sowie $§ 26$ Abs. 1 Satz 1 und Abs. 2 BDSG bei der Verarbeitung besonderer Kategorien personenbezogener Daten und der Datenverarbeitung im Beschäftigungskontext, aus dem Arbeitsvertrag oder bestehenden Betriebsvereinbarungen im Einsatzunternehmen. Zudem gibt die Verordnung explizit vor, Datenschutz durch Technikgestaltung und datenschutzfreundliche Voreinstellung vorzusehen. Gemäß Art. 25 Abs. 1 DSGVO muss der für die Datenverarbeitung Verantwortliche geeignete technische und organisatorische Maßnahmen (TOMs) treffen, um die Datenschutzgrundsätze aus Art. 5 Abs. 1 DSGVO wirksam umzusetzen. Dabei sind unter anderem die unterschiedlichen Eintrittswahrscheinlichkeiten und die Schwere der mit der Verarbeitung verbundenen Risiken für die Rechte und Freiheiten natürlicher Personen zu beachten. Zudem müssen die Maßnahmen gemäß Art. 5 Abs. 1 lit. f DSGVO eine angemessene Sicherheit für die verarbeiteten personenbezogenen Daten gewährleisten. Des Weiteren ist sicherzustellen, dass durch Voreinstellungen nur personenbezogene Daten, deren Verarbeitung für den jeweiligen bestimmten Verarbeitungszweck erforderlich ist, verarbeitet werden. So können nur solche Informationen über den einzelnen Beschäftigten hierzu als geeignet angesehen werden, die einen Beitrag für die Zwecke 
der Erreichbarkeitssteuerung liefern. Den ermittelten Risiken wurde durch das Einhalten rechtlicher Kriterien (z. B. Datenminimierung, Zweckbestimmung und Vertraulichkeit) entgegengewirkt, die im Projekt aus den rechtlicher Anforderungen (z. B. informationelle Selbstbestimmung und Systemintegrität) abgeleitet wurden (s. hierzu [7, 8]).

Anhaltspunkte für die Maßnahmenentwicklung bieten die Art. 25 und 32 DSGVO sowie der Stand der Technik. Dabei ist zu berücksichtigen, welches Risiko eines unbefugten Zugriffs auf die Daten prognostiziert wird, welches Ausmaß ein solches Ereignis annehmen kann und welche Daten durch das System verarbeitet werden [9]. So fordert Art. 32 Abs. 1 HS 2 lit. a und lit. b DSGVO TOMs, die die Vertraulichkeit und Integrität im Zusammenhang mit der Verarbeitung personenbezogener Daten auf Dauer sicherstellen, sowie die Verschlüsselung personenbezogener Daten, um ein den Risiken angemessenes Schutzniveau zu ermöglichen. Daraus wurden im Projekt technische Ziele und Gestaltungsvorschläge konkretisiert (s. hierzu [7]), die im EMS beispielsweise durch das Implementieren von Zugriffsrechten, Authentifizierungsverfahren, Verschlüsselungssoftware und kryptografisch sicheren Hashfunktionen umgesetzt wurden.

Durch den Einsatz des EMS im Arbeitskontext, wurden neben datenschutzrechtlicher Vorgaben zum anderen auch arbeitsrechtliche Bestimmungen berücksichtigt wie die des Arbeitszeitgesetzes (ArbZG) sowie des Betriebsverfassungsgesetzes (BetrVG). Hierzu gehören vor allem die Mitbestimmungsrechte des Betriebsrats gemäß $\S 87$ Abs. 1 Nr. 1 und 6 BetrVG bei der Systemeinführung im Einsatzunternehmen sowie die Vorgaben zur Arbeitszeit aus $\S 3$ ff. ArbZG.

\subsubsection{Stakeholder-Präferenzen}

Das Ziel unserer Forschung ist die Einführung eines verbesserten Erreichbarkeitsmanagements für Unternehmen und ihre Mitarbeiter. Hierdurch soll ermöglicht werden, die Vorteile moderner Informations- und Kommunikationstechnologien zu nutzen und entsprechenden Risiken vorzubeugen. Um dies zu erreichen, ist es essenziell, die Bedürfnisse und Ansprüche möglicher Nutzer zu kennen und zu verstehen [12]. Wir begannen mit der Definition des spezifischen Forschungsproblems und insbesondere mit der Frage, weshalb die bestehenden technologischen Lösungen nicht den Erreichbarkeitspräferenzen und -bedürfnissen der Benutzer gerecht werden. Es erfolgte eine Recherche über bestehende Applikationen, Geschäftslösungen und deren Limitierungen. Danach haben wir die Ziele eines SAA definiert, welcher darauf abzielt, die Komplexität der Präferenzen der Nutzer abzubilden. Dies wurde anhand der Analyse von Daten aus einer explorativen Interviewstudie $(\mathrm{N}=21)$ zum Thema intelligentes Erreichbarkeitsmanagements durchgeführt, um potenziell wichtige Inhalte und Ideen zu identifizieren [10]. Auf Basis dieses qualitativen Ansatzes fand eine große quantitative Benutzerumfrage mit 821 Teilnehmern statt [11]. Ziel war es dabei, einen möglichst breiten und umfassenden Einblick in die Bedürfnisse und Wünsche relevanter Stakeholder-Gruppen 
$\mathrm{zu}$ erhalten. Bezogen auf gewünschte Eigenschaften zeichnen sich fünf thematische Schwerpunkte ab: So soll der Erreichbarkeitsassistent sowohl individuell anpassbar sein als auch über Standardeinstellungen verfügen, die die Bedienung erleichtern. Bezüglich seiner Funktionsweise soll er als ein Filter fungieren, der Textnachrichten und Anrufe nach unterschiedlichen Kriterien weiterverarbeiten kann. Dabei soll er auch in der Lage sein, Kontakt mit dem Absender der Nachricht aufzunehmen und ein Feedback zu senden oder Abfragen zu tätigen. Ein Teil der Befragten wünscht sich zudem eine lernfähige und integrative Anwendung. Darüber hinaus äußerten fast alle Befragten den Wunsch, dass der Assistent möglichst unkompliziert und intuitiv verständlich sein und technisch einwandfrei funktionieren solle.

Die Analyse beider Studien lieferte eine Vielzahl an Erkenntnissen, welche die Entwicklung eines Erreichbarkeitsassistenten ermöglichen, der das Erreichbarkeitsmanagement der Mitarbeiter nachhaltig und effizient verbessert.

Einerseits bieten unsere Studienergebnisse hinsichtlich der Erweiterung der Grenztheorie [5] wertvolle Erkenntnisse darüber, wie Mitarbeiter ihren individuellen Grenzstil abstrahieren und in Erreichbarkeitspräferenzen übersetzen. Die Ergebnisse zeigen eindeutig, dass es kein festes Modell für die optimale Trennung und Integration von Arbeit und Privatleben gibt. Die meisten Teilnehmer wünschen entweder eine vollständige Trennung (37,3\%) oder eine interaktive Integration (35,7\%) von Beruf und Privatleben. Die gewünschten Erreichbarkeitszustände unterscheiden sich jedoch häufig von den tatsächlichen Zuständen. So erreichte nur die Hälfte der Befürworter einer vollständigen Trennung in der Praxis. Jeder fünfte Teilnehmer (19.7\%) äußerte im Gegensatz dazu berufliche Kontaktaufnahmen (Anrufe, E-Mails, SMS oder Messangernachrichten) außerhalb der Arbeitszeit, während nur 3,8\% der Teilnehmer dies wirklich wünschen. Insgesamt ist die Diskrepanz zwischen tatsächlicher und gewünschter Trennung und Integration von Arbeit und Privatleben (berechnet aus dem Vergleich des angegebenen tatsächlichen Stands der Grenzziehung zwischen Arbeit und Privatleben des Teilnehmers mit seiner gewünschten Erreichbarkeit bezüglich Arbeits- und Privatlebensgrenze) beträchtlich: Jeder zweite Teilnehmer (50,3\%) erreicht in der Praxis nicht seinen präferierten Zustand für eine Trennung und Verknüpfung von Arbeit und Privatleben [11].

Zum anderen, zeigen die Ergebnisse, dass die technologische Lösung, die ein differenziertes adaptives Management ermöglicht, über das Potenzial verfügt, zum Wohlbefinden des Einzelnen beizutragen. Die Praxis zeigt, dass aktuelle Arbeitgeber nicht ausreichend auf die unterschiedlichen Bedürfnisse ihrer Mitarbeiter eingehen, da sich die gegenwärtigen Lösungen nur auf die Einschränkung der Erreichbarkeit konzentrieren und so die Vielfalt unterschiedlicher Erreichbarkeitspräferenzen der Mitarbeiter nicht berücksichtigen. In diesem Zusammenhang besteht Bedarf nach einer technologischen Lösung, die die Komplexität der Erreichbarkeitspräferenzen der Menschen widerspiegelt. Unter diesen Umständen weist der Erreichbarkeitsassistent ein großes Potenzial für die erfolgreiche Verwaltung und Regulierung der Erreichbarkeit von Einzelpersonen auf. Er unterstützt die Flexibilität und Autonomie der Benutzer und geht auf individuelle Wünsche und Bedürfnisse ein. Insbesondere die Mehrheit 
der Studienteilnehmer (55,9\%) validiert das zugrundeliegende Konzept des Smarten Assistenten für Erreichbarkeit, da sie ihn als nützlich erachtet und einer Verwendung des Assitenten zustimmt [11]. Darüber hinaus zeigt unsere Analyse, dass diese Art von Assistenten insbesondere Mitarbeiter mit einem Missverhältnis zwischen ihrem tatsächlichen und ihrer bevorzugten Grenzziehung zwischen Arbeit und Privatleben unterstützt. Führungsverantwortung; von großen Unternehmen und/oder Personen, die in der Freizeit berufsbezogene Anrufe erhalten. Im Unterschied dazu zeigen die Ergebnisse, dass ältere Mitarbeiter diese Technologielösung seltener einsetzen. Es zeigt den Bedarf an mehr Information und Bewusstsein für intelligentes Erreichbarkeitsmanagement sowie Schulungskonzepten und organisatorische Maßnahmen für Mitarbeiter. Diese werden in dem nächsten Kapitel betrachtet.

\subsubsection{Organisatorische Maßnahmen}

Der Maßnahmenkatalog liefert konkrete Ansatzpunkte und Rahmenbedingungen, um das Erreichbarkeitsmanagement sinnvoll zu gestalten und zu fördern. Die organisatorischen Maßnahmen umfassen zum einen allgemeine Empfehlungen und zum anderen konkrete Maßnahmen für den Beschäftigten sowie auch für den Arbeitgeber. Darüber hinaus beinhaltet der Maßnahmenkatalog Empfehlungen für die Funktionsweisen des Erreichbarkeitsassistenten.

Hinsichtlich der allgemeinen Empfehlungen können die folgenden Punkte festgehalten werden:

\section{Schaffung eines stärkeren Bewusstseins für das Erreichbarkeitsmanagement}

Anhand der Ergebnisse war ein Missmatch zwischen tatsächlicher und gewünschter Erreichbarkeit in der Freizeit ersichtlich. Das Erreichbarkeitsmanagement sollte daher überall eine Rolle spielen, wo moderne Kommunikationstechnologien im Einsatz sind.

\section{Steigerung der Kommunikation über Erreichbarkeit und Erreichbarkeitsverhalten} Bezüglich der Erwartungen an die Erreichbarkeit war eine kommunikative Lücke erkennbar. Diese sollte geschlossen werden, um ein erfolgreiches Erreichbarkeitsmanagement zu ermöglichen

Förderung der Möglichkeit eines individuell passenden Erreichbarkeitsmanagements Da eine Pauschallösung für alle Beschäftigten nicht sinnvoll ist, sollten Erreichbarkeitslösungen individuelle Präferenzen einzelner Beschäftigter berücksichtigen.

Aus der Sicht des Arbeitnehmers lassen sich folgende Maßnahmen ableiten:

\section{Bewusstmachen der Bedeutung von Erreichbarkeitsmanagement}

Um die Bedeutung der Erreichbarkeit hervorzuheben, können Informationsmaterial sowie Informationsveranstaltungen und Schulungen genutzt werden. Es sollten die Problematik der ständigen Erreichbarkeit sowie auch die Vorteile der flexiblen Arbeits- 
welt thematisiert werden. Das Kennen der eigenen Erreichbarkeitspräferenzen stellt einen weiteren wichtigen Bereich dar.

Für Führungskräfte ist darüber hinaus die Bedeutung der offenen Kommunikation über das Erreichbarkeitsmanagement sowie auch die Rolle des eigenen Erreichbarkeitsverhaltens zentral.

\section{Reflexion des eigenen Erreichbarkeitsverhaltens}

Mithilfe von Reflexionsmaterial sowie im Rahmen von Schulungen sollte eine Reflexion über das präferierte und das tatsächliche Erreichbarkeitsverhalten sowie die daraus resultierende erlebte Beanspruchung erfolgen. In diesem Zuge sollten auch verbesserte Erreichbarkeitslösungen überdacht werden.

\section{Bemühung um eine individuell passende Erreichbarkeitslösung}

Hierbei ist eine Vereinbarung zur individuellen Erreichbarkeit mit der Führungskraft und dem Team sowie eine entsprechende aktive Anpassung des eigenen Verhaltens empfehlenswert.

Für ein optimales Erreichbarkeitsmanagement des Beschäftigten ist der Rückhalt durch den Arbeitgeber zentral. Daher gelten folgende Empfehlungen für den Arbeitgeber:

Das Erreichbarkeitsmanagement sollte ein Gesundheitsthema im Unternehmen sein Der Arbeitgeber sollte Informations- und Reflexionsmaterial anbieten. Darüber hinaus ist das Bereitstellen eines Erreichbarkeitsassistenten empfehlenswert. Durch das Anregen von Gesprächsrunden sowie Mitarbeiterversammlungen sollte zur Nutzung des Materials motiviert werden.

\section{Festlegen von Standards bezüglich des Erreichbarkeitsmanagements im Unter- nehmen}

Durch das Festlegen von Standards soll Transparenz geschaffen werden. Das Festhalten von Zeiten für (eingeschränkte) Erreichbarkeit und Nicht-Erreichbarkeit kann im Rahmen von Betriebsvereinbarungen festgehalten werden.

Individuelle Erreichbarkeitslösungen sollten zudem im Rahmen verbindlicher Einzelgespräche regelmäßig festgehalten werden. Zu klärende Punkte sind hierbei, unter welchen Umständen, über welche Kanäle, zu welchen Zeiten eine Erreichbarkeit außerhalb der Arbeitszeit besteht.

\section{Förderung einer langfristigen Wirkung des Erreichbarkeitsmanagements im Unter- nehmen}

Zuletzt wird die Durchführung von Qualitätskontrollen und Maßnahmenevaluationen empfohlen. Dies kann im Zuge von Mitarbeiterbefragungen sowie durch das Einholen von Verbesserungsvorschlägen in Mitarbeiterversammlungen erfolgen. Entsprechendes Feedback sollte umgesetzt werden. 
Als Empfehlungen für die Funktionsweisen des Erreichbarkeitsassistenten ergaben sich die folgenden Punkte:

\section{Möglichkeit zur individuellen Anpassung}

Der Erreichbarkeitsassistent sollte eine Anpassung an die Bedürfnisse und Arbeitsumstände einzelner Beschäftigter erlauben.

\section{Einfache Bedienbarkeit und Standardeinstellungen}

Der Erreichbarkeitsassistent sollte einfach und intuitiv bedienbar sein. Für eine beschleunigte Nutzung sollte er darüber hinaus über (individuell anpassbare) Standardeinstellungen verfügen.

\section{Unterscheidung nach Kommunikationskanälen, Inhalten und Personengruppen}

Der Erreichbarkeitsassistent sollte die Möglichkeit anbieten, nur per Textnachricht/EMail erreichbar zu sein, da dies oftmals als nützlich und weniger störend empfunden wurde.

Darüber hinaus sollte die Möglichkeit der Erreichbarkeit nur in Notfällen bestehen, wobei private Notfälle hier besonders zu gewichten sind.

Zuletzt ist eine Option empfehlenswert, die die Erreichbarkeit für selbstgewählte Personengruppen beinhaltet.

\section{Informieren des Adressaten über Verzögerung und Wiedererreichbarkeit}

Der Absender sollte durch den Erreichbarkeitsassistenten über die Verzögerung sowie die Wiedererreichbarkeit informiert werden.

Zudem ist eine kontinuierliche Verbesserung des Erreichbarkeitsassistenten über Feedback der Anwender notwendig.

\subsubsection{Erreichbarkeitsassistent für Smartphones}

Neben den organisatorischen Maßnahmen wurde im Projekt außerdem ein Erreichbarkeitsassistent in Form eines Demonstrators für die Smartphone-Plattform Android umgesetzt. Dieser ermöglicht die automatische Verwaltung der Erreichbarkeit des Nutzers über elektronische Kommunikationswege wie E-Mail und Telefonanrufe und besteht aus zwei getrennten Teilen. Einer Smartphone-App, die es dem Nutzer erlaubt individuelle Anpassungen seiner Erreichbarkeit vorzunehmen und in der Lage ist eingehende Telefonanrufe automatisch abzuweisen, sowie einer zentralen, serverseitigen Komponente für die Verwaltung dieser Einstellungen und die Verarbeitung von E-Mails.

Die zentrale Behandlung der E-Mails erlaubt es, die Kommunikationsregeln unabhängig des verwendeten E-Mail-Programms umzusetzen. Während einige Programme bereits über Möglichkeiten verfügen, bestimmte Regeln zur Erreichbarkeit 
zu definieren, unterscheiden diese sich zwischen Programmen und Plattformen (z. B. Desktop oder Smartphone), sodass es nicht möglich ist, die Kommunikationsregeln identisch auf allen Geräten umzusetzen. Da diese Komponente außerdem die Aufgabe des bisherigen Mailservers übernimmt, ist auf Benutzerseite lediglich eine Einstellung des Benutzerkontos für die Einführung des Systems notwendig.

Durch die Definition von Regeln kann individuell entsprechend der Anforderungen im Unternehmen mit einem Regeleditor die konkrete Funktionalität des Erreichbarkeitsassistenten konfiguriert werden, etwa im Rahmen einer Verhandlung zwischen Arbeitgeber und Betriebsrat. So können z. B. dienstliche E-Mails oder Telefonate in der Nacht entsprechend des Regelwerks unterdrückt bzw. zu einem späteren Zeitpunkt zugestellt werden. Für die Entscheidung, ob Kommunikation zugelassen, unterbunden oder verzögert wird, können je nach Verfügbarkeit im jeweiligen Unternehmen diverse Kriterien herangezogen werden. In der ersten Evaluationsphase des Pilotbetriebs wurde mit den Anwendungs-Unternehmen zuerst ein simples und starres Regelwerk im Erreichbarkeitsmanager erarbeitet und umgesetzt. Das umgesetzte Regelwerk bildet z. B. die üblichen Geschäftszeiten in den beiden Anwender-Unternehmen ab.

In diesem Regelwerk wird zwischen drei Erreichbarkeitsstufen unterschieden:

1. Volle und uneingeschränkte Erreichbarkeit während der gesamten Kernarbeitszeit

2. Eingeschränkte Erreichbarkeit für bestimmte Kontakte

3. Störungsfreie Nachtruhe

Bei voller Erreichbarkeit werden alle E-Mails sofort und ohne Verzögerung zugestellt. Während der Nachtruhe werden alle eingehenden E-Mails bis zum nächsten Morgen zurückgehalten und, abhängig von der Sendeadresse, entweder bei Beginn der eingeschränkten Erreichbarkeit, spätestens aber mit Beginn der Kernarbeitszeit zugestellt.

Die eingeschränkte Erreichbarkeit bildet im Falle eines Anwenderunternehmens den Anwendungsfall ab, außerhalb der Kernarbeitszeiten nur für bestimmte Kontakte erreichbar zu sein. Im konkreten Fall sind das internationale Geschäftspartner für welche, durch ihren Sitz im Ausland und der daher auftretenden Zeitverschiebung, durch diese Erreichbarkeitsregeln ein realistischeres Kommunikationsfenster geschaffen wird. Eingehende Kommunikation dieser Geschäftskontakte wird während der eingeschränkten Erreichbarkeit ohne Verzögerung zugestellt, E-Mails von Absendern außerhalb dieser Ausnahmen werden, wie zur Nachtruhenzeit, bis zum Beginn des nächsten Geschäftstages zurückgehalten.

In der nächsten Evaluationsphase wurde es mit Einführung der SmarthoneApplikation dem Nutzer ermöglicht, das, in der vorherigen Phase eingeführte, Regelwerk zu personalisieren. Dazu wurde die Möglichkeit geschaffen, die Anfangs- und Endzeiten der jeweiligen Erreichbarkeitsstufen zu wählen. Die Verwaltung der telefonischen Erreichbarkeit muss dahingegen dezentral auf dem jeweiligen Endgerät erfolgen, da hier keine verwaltbare, zentrale Komponente existiert. Zudem ist es auf Android und iOS, 
den am weitesten verbreiteten Smartphone-Betriebssystemen ${ }^{1}$, nicht möglich alle eingehenden Anrufe zu blockieren und nur bestimmte Nummern zu erlauben (Whitelisting). Stattdessen ist es nur möglich bestimmte Nummern automatisch abzuweisen (Blacklisting).

Der Entwickelte Demonstrator bietet dem Nutzer folgende Funktionalität:

- Verwaltung von Erreichbarkeitsprofilen, welche zeitbasiert aktiviert werden und es dem Nutzer so erlauben, verschiedene Funktionen und Einstellungen des Systems automatisch zu aktivieren.

- manueller und temporärer Wechsel des aktuell aktivierten Profils

- Automatischer Wechsel des Profils basierend auf Kalendereinträgen (z. B. eingeschränkte Erreichbarkeit im Urlaub/während Meetings)

- Zurückhalten von E-Mails bis zu einem gewissen Zeitpunkt oder Wechsel in ein unterschiedliches Profil

- Gebündelte Zustellung von E-Mails (z. B. zu jeder vollen Stunde) um „Fokusphasen“ ohne ablenkende Kommunikation zu erlauben

- Blockieren von eingehenden Anrufen auf Nummernbasis

Die Einstellung dieser Funktionen erfolgt über die entwickelte Smartphone-App oder ein Webinterface (Abb. 23.1).

\subsubsection{Testbetrieb und Evaluation}

Für den Testbetrieb bei den Anwender-Unternehmen und die Evaluation der technischen Maßnahmen muss, wie im vorigen Abschnitt beschrieben, die zentrale Komponente für die Verwaltung der Einstellungen sowie die E-Mail-Kommunikation dem Anwender bereitgestellt werden. Da die zentrale Komponente für jeden Anwender (jedes Unternehmen, nicht jeder Mitarbeiter) speziell konfiguriert werden muss sowie zur Sicherstellung des Datenschutzes, wird das System für jeden Anwender getrennt betrieben. Zur Einhaltung der Rechtskonformität nach der Datenschutz-Grundverordnung wird außerdem sichergestellt, dass jedes System aus einem in Deutschland betriebenen Rechenzentrum bereitgestellt wird.

Im Forschungsprojekt SANDRA wird die HRV-Messung eingesetzt, um die Auswirkung des intelligenten Erreichbarkeitsmanagements auf die Erholung während des nächtlichen Schlafs zu messen. Akuter und chronischer Stress, insbesondere auch der durch Arbeit hervorgerufene chronische Stress, wirken sich vor allem auf das Autonome Nervensystem (ANS) aus [16, 17]. Messbar sind die stressbedingten Veränderungen des ANS über die Herzratenvariabilität (HRV) [18]. Generell müssen für die Bestimmung

\footnotetext{
${ }^{1}$ https://gs.statcounter.com/os-market-share/mobile/worldwide/\#monthly-201901-201912
} 


\section{$11: 57 \widehat{\mathrm{S}} \boldsymbol{\nabla}$ 口全}

Profileinstellungen für: Arbeit

Allgemein

Start

Bestimmt ab wann das Profil gültig ist

Ende

Bestimmt den Endzeitpunkt der Profilgültigkeit

(1) Kalendertermine

Das automatische umstellen von Profilen

basierend auf Kalendereinträgen ist deaktiviert.

Eintrag

Name des Kalendereintrags der den Profilwechel auslöst.

Profil in das gewechselt werden soll.

Nicht festgelegt

\section{Gebündelte Zustellung}

Die gebündelte Zustellung ist aktiviert

\section{Zustellungsintervall}

Bestimmt die Zeit ziwschen den einzelnen 60 Minuten Zustellungen

\section{Zustellungsversatz}

Bestimmt wann die erste Zustellung nach O Minuten aktivierung des Profils erfolgen soll

\section{․ Blockierte Telefonnummern}

Eingehende Anrufe von den Telefonnummern in dieser $\mathrm{L}$...

Abb. 23.1 Screenshot der App zur Verwaltung der Nutzereinstellungen 
der HRV-Parameter die RR-Abstände zwischen den Herzschlägen gemessen werden. Für diese Messung braucht es aber keine teuren EKG-Geräte, sondern es reichen handelsübliche Brustgurte, z. B. der Firma Polar, um diese EKG-genau zu messen. Die Analyse der HRV ermöglicht eine Früherkennung pathogener Zustände.

Für die Evaluation der Stressminderung wurde im Rahmen des Projekts eine Smartphone-App zur Messung der HRV-Parameter implementiert. Die App empfängt die vom Brustgurt aufgenommenen RR-Intervalle über Bluetooth. Die Messung wird von der Smartphone-App an einen Server übertragen. Dort werden die HRV-Parameter berechnet. Ihr zeitlicher Verlauf kann über den Browser analysiert werden. Die Hypothese ist, dass sich durch den Verzicht auf berufliche Kommunikation nach Feierabend, die HRV früher dem Erholungspegel annähert als im gegenteiligen Fall. Dazu wurden bei Mitarbeitern der an der Evaluation teilnehmenden Partnerunternehmen unter beiden Prämissen - mit und ohne berufliche Korrespondenz am Abend - HRV-Messungen über die Nacht durchgeführt. Dafür sollte die Messung beim Zubettgehen gestartet und am nächsten Morgen unmittelbar nach Erwachen beendet werden. Nach dem Hochladen der Messung auf den Server und der Berechnung der HRV Parameter, kann diese über den Webbrowser analysiert werden.

Die HRV-Parameter nehmen nicht nur durch Belastung bzw. Stress am Vorabend niedrige Werte an, sondern auch durch

- Bewegung während der Messung

- Husten und Nießen während der Messung (temporär)

- Alkoholkonsum vor der Messung

- Üppiges und spätes Essen vor der Messung

- Körperliche Belastung (Sport) in den Stunden vor der Messung

- Erkältungen, grippale Effekte und andere Krankheiten

- Medikamenteneinnahme

Für die Studie im Rahmen des SANDRA Projektes ergibt sich daraus die Rahmenbedingung, an den Messtagen die oben genannten Faktoren auszuschließen bzw. konstant zu halten, sodass die variierende geschäftliche Email-Kommunikation am Abend als einzige Einflussgröße angenommen werden kann.

\subsection{Fazit und Ausblick}

Im Forschungsprojekt SANDRA wurden sowohl ein Erreichbarkeitsassistent als auch organisatorische Maßnahmen in zwei Anwender-Unternehmen aus zwei verschiedenen Branchen erprobt. Die bisherigen Erkenntnisse aus dem Testbetrieb deuten darauf hin, dass sich durch eine Mischung technischer und organisatorischer Ansätze zur Verringerung der Belastung durch ständige Erreichbarkeit über Smartphones erreichen lassen [4]. 
Das Zusammenspiel zwischen Recht und Technik ist maßgeblich, um der schnellen Entwicklung neuer technischer Innovationen zu begegnen, da sie zahlreiche Bereiche des gesellschaftlichen Lebens beeinflussen und verändern. Sie werden in unterschiedlichen gesellschaftlichen Bereichen zu bestimmten Zwecken und zur Erfüllung bestimmter Ziele eingesetzt. Die Sicherheit von Daten und der Schutz der von der Verarbeitung ihrer Daten betroffenen Personen ist maßgeblich von der einzelnen Technologie abhängig. Entstehen diesbezügliche aus ihrer Verwendung Probleme, werden sie meist erst nach einem gewissen Nutzungszeitraum erkannt und sollen durch das nachträgliche Einbauen von Schutzmechanismen behoben werden. Eine viel praxistauglichere Lösung bietet die rechtliche Gestaltung eines technischen Systems, die begleitend zur Technikentwicklung oder bereits in einer konzeptionellen Phase der Entwicklung berücksichtigt wird. Diese Vorgehensweisen stellen sicher, dass der Einsatz der Technik nicht an rechtlichen Hürden scheitert oder Kosten im Nachgang, aufgrund einer nicht rechtskonformen Bauweise, vermieden werden. Zudem ist ein technischer Schutz von personenbezogenen Daten oder Unternehmensdaten sehr effektiv, denn wenn etwas technisch nicht möglich ist, kann ein darauf bezogenes rechtliches Verbot nur schwer umgangen werden.

Für die Zukunft stellt sich die Frage, ob weitere Kommunikationstechnologien in ein Erreichbarkeitsmanagement einzubeziehen sind. Hierbei relevant erscheinen aufgrund ihrer gestiegenen Verbreitung im Umfeld dienstlicher Kommunikation, z. B. Instant Messaging Systeme oder auch Varianten aus den Social Media - Umfeld.

\section{Projektpartner und Aufgaben}

- Fraunhofer-Institut für Arbeitswirtschaft und Organisation IAO Identitätsmanagement, Konzeption des Erreichbarkeitsmanagements, Projektkoordination

- Universität Kassel - Projektgruppe verfassungsverträgliche Technikgestaltung (provet)

Rechtsverträgliche Gestaltung eines Erreichbarkeitsmanagement-Systems

- HdM Stuttgart - Mobile Medien

Entwurf und Implementierung des Erreichbarkeitsmanagers

- TU Darmstadt - Fachgebiet Marketing \& Personalmanagement State of the Art und Stakeholderanforderungen für das Erreichbarkeitsmanagement

- Goethe Universität-Frankfurt - Wirtschaftsinformatik und Informationsmanagement (WIIM)

Quantitative Analyse der Stakeholderpräferenzen für Erreichbarkeitsmanagement-Systeme

- AGILeVIA GmbH

Implementierung im Unternehmen und Erprobung 


\section{- AK Reprotechnik GmbH}

Mitgestaltung des Erreichbarkeitsmanagements aus Anwendersicht und Erprobung

\section{Literatur}

1. Ayyagari R, Grover V, Purvis R (2011) Technostress: Technological antecedents and implications. MIS Q 35(4):831-858

2. David K, Bieling G, Jandt S, Ohly S, Roßnagel A, Schmitt A, Steinmetz R, Stock-Homburg R, Wacker A (2014) Balancing the online life: mobile usage scenarios and strategies for a new communication paradigm. IEEE Veh Technol Mag, September, S 72-79

3. Fonner KL, Stache LC (2012) All in a day's work, at home: Teleworkers'management of micro role transitionsand the work-home boundary. New Technol Work Employ 27(3):242257

4. Grießhaber D, Maucher J, Laufs U, Staab K, Saternus Z, Weinhardt S. (2020) Erreichbarkeitsmanagement in der betrieblichen Praxis. In: Daum M, Wedel M, Zinke-Wehlmann C, Ulbrich $\mathrm{H}$ (Hrsg) Gestaltung vernetzt-flexibler Arbeit. Beiträge aus Theorie und Praxis für die digitale Arbeitswelt. Springer Vieweg, Berlin, i. E.

5. Kossek EE (2016) Managing work-life boundaries . Organ Dyn 45:258-270

6. Laufs A, Maucher J, Miedzianowski N, Rost K, Saternus Z (2018) Erste Ergebnisse des Forschungsprojekts „SANDRA“. Zeitschrift für Datenschutz-Aktuell (10/2018): 06151

7. Miedzianowski N (2020) Rechtliche Anforderungen an ein System zur Erreichbarkeitssteuerung. In: Daum M, Wedel M, Zinke-Wehlmann C, Ulbrich H (Hrsg) Gestaltung vernetztflexibler Arbeit. Beiträge aus Theorie und Praxis für die digitale Arbeitswelt, Springer Vieweg, Berlin, i. E.

8. Miedzianowski N, Saternus Z, Staab K (2019) Stakeholderbezogene und rechtliche Anforderungen an ein Erreichbarkeitsmanagement-System. Zeitschrift für Datenschutz (11/2019): XV--XIX

9. Reimer P (2018) DSGVO Art. 5 Grundsätze für die Verarbeitung personenbezogener Daten. In: Sydow G (Hrsg) Europäische Datenschutzgrundverordnung. Nomos, Baden-Baden, Wien, Zürich, Art. 5 Rn. 52

10. Saternus Z, Staab K (2018) Towards a smart availability assistant for desired work life balance. International Conference on Information Systems 2018, San Francisco, USA

11. Saternus Z, Staab K, Hinz O (2019) Challenges for a Smart Availability Assistant Availability Preferences. Americas Conference on Information Systems 2019, Cancun, Mexiko

12. Saternus Z, Staab K, Hinz O, Stock-Homburg R (2020) Ein nutzergerechtes Erreichbarkeitsmanagement: Wissenschaftliche Erkenntnisse und Implikationen. In: Daum M, Wedel M, Zinke-Wehlmann C, Ulbrich H (Hrsg) Gestaltung vernetzt-flexibler Arbeit. Beiträge aus Theorie und Praxis für die digitale Arbeitswelt. Springer Vieweg, Berlin

13. Sayah S (2013) Managing work-life boundaries with information and communication technologies: the case of independent contractors. New Technol Work Employ 28(3):179-196

14. Stock-Homburg R, Bieling G, Entringer T, Reinke K (2014) New directions for worklife balance research: a conceptual, qualitative approach, Proceedings of the Academy of Management Conference, Philadelphia, USA 
15. Tarafdar M, Tu Q, Ragu-Nathan BS, Ragu-Nathan TS (2007) The impact of tech-nostress on role stress and productivity. J Manage Inf Syst 24(1):301-328

16. Henry J (1997) Psychological and physiological responses to stress: the right hemisphere and the hypothalamo-pituitary-adrenal axis, an inquiry into problems of human bonding. Acta Physiol Scand Suppl 640:10-25

17. Schroeder EB, Liao D, Chambless LE et al (2003) Hypertension, blood pressure, and heart rate variability: the atherosclerosis risk in communities (ARIC) study. Hypertension 42:11061111. https://doi.org/10.1161/01.HYP.0000100444.71069.73

18. Togo F, Takahashi M (2009) Heart Sew. Ind Health 47:589-602. https://doi.org/10.2486/ indhealth.47.589

Open Access Dieses Kapitel wird unter der Creative Commons Namensnennung 4.0 International Lizenz (http://creativecommons.org/licenses/by/4.0/deed.de) veröffentlicht, welche die Nutzung, Vervielfältigung, Bearbeitung, Verbreitung und Wiedergabe in jeglichem Medium und Format erlaubt, sofern Sie den/die ursprünglichen Autor(en) und die Quelle ordnungsgemäß nennen, einen Link zur Creative Commons Lizenz beifügen und angeben, ob Änderungen vorgenommen wurden.

Die in diesem Kapitel enthaltenen Bilder und sonstiges Drittmaterial unterliegen ebenfalls der genannten Creative Commons Lizenz, sofern sich aus der Abbildungslegende nichts anderes ergibt. Sofern das betreffende Material nicht unter der genannten Creative Commons Lizenz steht und die betreffende Handlung nicht nach gesetzlichen Vorschriften erlaubt ist, ist für die oben aufgeführten Weiterverwendungen des Materials die Einwilligung des jeweiligen Rechteinhabers einzuholen.

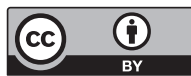

